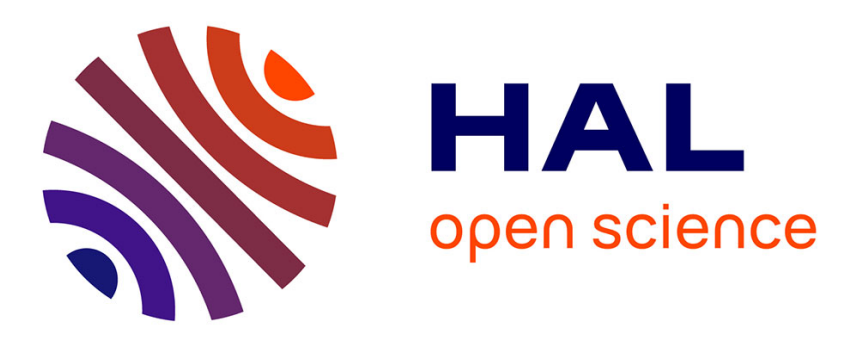

\title{
Investigation of the internal structure of hemp fibres using optical coherence tomography and focused ion beam transverse cutting
}

Vincent Placet, Jérémy Meteau, Luc Froehly, Roland Salut, Lamine Boubakar

\section{- To cite this version:}

Vincent Placet, Jérémy Meteau, Luc Froehly, Roland Salut, Lamine Boubakar. Investigation of the internal structure of hemp fibres using optical coherence tomography and focused ion beam transverse cutting. Journal of Materials Science, 2014, 49 (24), pp.8317 - 8327. hal-02300211

\section{HAL Id: hal-02300211 \\ https://hal.science/hal-02300211}

Submitted on 29 Sep 2019

HAL is a multi-disciplinary open access archive for the deposit and dissemination of scientific research documents, whether they are published or not. The documents may come from teaching and research institutions in France or abroad, or from public or private research centers.
L'archive ouverte pluridisciplinaire HAL, est destinée au dépôt et à la diffusion de documents scientifiques de niveau recherche, publiés ou non, émanant des établissements d'enseignement et de recherche français ou étrangers, des laboratoires publics ou privés. 


\title{
Investigation of the internal structure of hemp fibres using Optical Coherence Tomography and Focused Ion Beam transverse cutting
}

\author{
Vincent Placet ${ }^{1}$, Jérémy Méteau ${ }^{2}$, Luc Froehly ${ }^{2}$, Roland Salut ${ }^{2}$, M. Lamine Boubakar ${ }^{1}$ \\ FEMTO-ST Institute, UMR CNRS 6174, University of Franche-Comté, F-25000 Besançon, \\ ${ }^{l}$ Department of Applied Mechanics, \\ ${ }^{2}$ Department of Optics
}

Corresponding author: Email: vincent.placet@ univ-fcomte.fr - tel: +33 (0)3 81666055 - fax: +33(0)38166 6700

\begin{abstract}
The use of plant fibres in composite applications requires an efficient characterisation of their mechanical properties and thus an accurate description of their internal structure. The review of literature points out that there is still a lack of data on the organisation and structure of bast fibres. In this study, we propose to investigate the internal structure of hemp fibres using two experimental techniques: Focused Ion Beam (FIB) microscopy and Optical Coherence Tomography (OCT). Results indicate that OCT, a nondestructive and non-invasive technique, is a powerful technique to quickly and easily describe the internal structure of fibres and also to discriminate single fibres from bundle of fibres. In this paper, we also show that among technical hemp fibres and for a same range of external diameters (of about 20-30 microns), two types of internal structures can be observed: (i) elementary fibres with a thick wall and a small lumen and (ii) bundle of small fibres with an external diameter of a few microns. According to data of literature, these two structures were identified as being respectively primary fibres and bundle of secondary fibres. This result is of great importance for the mechanical characterization of the bast hemp fibres. Indeed, this means that during the test campaigns, the batch of isolated fibres is undoubtedly composed of both single primary fibres and bundle of secondary fibres. It certainly participates to the high scattering in results.
\end{abstract}

Key words: OCT/ FIB / hemp fibres / internal structure 


\section{Introduction}

In recent years, composite industries and researchers have shown a keen interest in plant-based fibres. However, before such fibres can be used for the reinforcement of organic matrices in high performance applications as an alternative to man-made fibres, an accurate understanding of their mechanical behaviour is required [1].

These fibres are effectively characterised by an intricate structure, morphology and organisation [2] which makes the determination of their mechanical properties more complex than for solid circular man-made fibres [3-4]. The elementary fibres have a typical cell plant structure, with a lumen and a particularly thick wall. They have a rounded polygonal outer shape, which is irregular and non-uniform along length of the fibre, and also varies from one fibre to another. The central cavity can be narrow, round or elliptical, with a diameter depending of the plant maturity [5]. As in other fibre-rich plants but contrary to flax, hemp produces in addition to the primary bast fibres, secondary fibres. These two types of fibre occurring in hemp show different and independent development. The primary fibres appear earlier in the development in the rapid growth stage and are formed from the primary meristem in the phloem. At maturity, primary fibres reach many millimetres in length (up to $100 \mathrm{~mm}$ ) and with compact cell walls that almost fill the lumen [5-7]. They are present from bottom to top of the plant $[5,8]$. In contrast, the secondary fibres are formed in later stages during thickness growth from the secondary meristem. Secondary fibres are generally thinner, shorter and with higher lignin content than the primary fibres. Figure 1 a-e, published in [8] and kindly provided by P. Knox with permission for use here, show the location, structure and morphology of these two fibre types. The proportion of primary fibres in the bast fibres is generally measured from 73 to $93 \%$ [9] and is showed to affect mean strength of fibres [10].

The variability in fibre properties, often pointed out in literature, can be ascribed either to the variability introduced by the applied experimental characterisation method, or to the inherent variability of the fibre themselves [11]. An important inaccuracy is especially achieved in the tensile testing procedure when determining the crosssectional area of the fibres. A typical approach consist of considering the fibres as being circular, ignoring the presence of the lumen, and using the measured fibre diameter to evaluate the cross-sectional area as for a cylinder. This is found to be one of the major reasons for the variability in properties [11]. Virk et al. [12] demonstrated for jute fibres 
that the coefficient of variation of the strain at failure (which is independent of the fibre cross-section unlike the modulus and strength), is consistently lower than that of strength or rigidity; lower value of $\mathrm{CoV}$ signifying smaller variations.

Another difficulty, when working with elementary fibres, is to ensure the single character of the fibre. This point is unfortunately only poorly discussed in literature. Effectively, in the stem of the plant, elementary fibres are glued together by a pectin interface, to form technical fibre bundles. Mechanical, chemical, or bacteria treatments, inducing a more or less important decomposition of the cell wall, can be used. The measured mechanical properties are highly dependent to the procedure used to extract the fibre from the plant. Burgert et al. proposed an interesting comparison of the properties of fibres chemically or mechanically isolated [13-15]. Bourmaud et al. [16] proposed for flax a water-washing treatment to facilitate the extraction of elementary fibre and to preserve the fibre integrity. So, whatever the method used to isolate the fibres, it is crucial to establish a rapid, non-destructive and objective method to observe the inner structure and 3-D shape of the fibre and ensure its unitarian character. This is a real challenge from an experimental point of view. The difficulty comes from the size and fragility of elementary fibres making preparation and handling challenging. Using optical microscopy, the lumen can be revealed looking at the fibre against the polarised light $[2,17]$. Unfortunately, the contrast between the cell wall and the lumen is sometimes inadequate and the cell wall transparency not enough to ensure a quantitative analysis. As underlined by Abbey et al. [18], the mode of visualisation is also important and may involve interaction between the probe and the fibre. SEM observations can, for example, induce deformation or shrinkage of a fibre, due to the use of low vacuum conditions, and produce significant surface modifications due to electron beam damage. Charlet and co-authors [19,20] proposed also direct insights on flax fibre structure by Focused Ion Beam (FIB) microscopy. In this case, the cell wall is damaged. Gallium ions strongly interact with the flax fibre releasing secondary ions and secondary electrons which are used to capture an image of the sample surface.

Observations of the fractured end surface after tensile test, as proposed by several authors, do not constitute a relevant method since significant modifications of the fibre geometry and damages could have occurred under mechanical loading. The observation can also be performed before the tensile test after cutting the fibre extremity. Regrettably, cutting techniques using microtome can induce damage and deformation. 
Clair et al. [21] observed on poplar tensionwood fibres a reduction of lumen area at the cutting level.

So, it is undoubtly recommended to use non-invasive, non-destructive techniques to characterise the fibre morphology. X-ray micro tomography is a powerfull and promising method to produce 3D digital representations of elementary fibres by mathematically reconstructing projection data collected by illuminating the fibre with $\mathrm{X}$-rays from many directions [18,22]. However, this technique requires high resolution synchrotron systems and is time consuming. This technique is inappropriate for a quick morphological characterisation as necessitated by tensile test campaigns. Optical methods constitute also promising methods. Malek et al. [23] showed interesting results using digital holographic tomography on flax fibres.

This work proposes a different optical tomography technique for rapid, accurate and non-destructive visualization of the inner structure of the fibres: the optical coherence tomography (OCT). OCT is a high resolution, non invasive and real time optical imaging technique that has attracted great interest over the last two decades especially in the field of biomedical imaging (ophthalmology, gastroenterology and cardiology). The technique can perform a cross-sectional tomographic imaging of the internal microstructure of materials and biological tissues [24,25]. It is a similar principle than this of echography except that it uses light instead of sound. This system measures the backscattered and backreflected light from the different layers in the sample. In terms of performance, compared to echography, OCT has a resolution of 10 to 100 times finer but the available depth is 10 times lower. The resolution is close to confocal microscopy (1 to $15 \mu \mathrm{m}$ ) with a penetration depth of $2-3 \mathrm{~mm}$, limited by scattering and absorption of the tissue. The principle of this technique was invented by J.G. Fujimoto in the late 1980s and the OCT imaging was demonstrated in 1991 by Huang et al [26]. The first commercial system (for ophtalmic diagnosis) has been proposed by Carl Zeiss Meditec in 1996.

Today OCT encounters impressive developments and improvements. There are two main configurations for OCT systems: Time Domain OCT (TDOCT) and Fourier Domain OCT (FDOCT), the later being the most powerful in term of detection capability. The potentiality of this technique was already proved for in vivo visualization of plant cells by Hettinger et al. [27,28] with a spatial resolution of $5 \mu \mathrm{m}$. This work proposes an investigation using an enhanced OCT technology, which is an 
hybrid system developed in the FEMTO-ST laboratory (Optical Department) for primarily a spectroscopic purpose with a spatial resolution of approximately $1 \mu \mathrm{m}$. A comparison between the internal structure revealed using FIB transverse cutting and OCT intensity profiles is proposed.

\section{Materials and methods}

\section{$2.1 \quad$ Plant material}

Hemp fibres (Cannabis sativa L.) were procured from the LCDA Company in France. Elementary fibres were isolated by hand and glued on thin paper frame to facilitate and simplify their handling.

\subsection{Optical Coherence Tomography}

The OCT technology used for this work is an hybrid system developed using scan free time correlator (Fig.2). This is an alternative to TDOCT and FDOCT [29-32] that was demonstrated at the end of the 90ies [33, 34] although the principle was already shown in 57 by Connes [35]. Such a system is a time domain correlator with a direct and instantaneous registration of the Amplitude modulation scan (Ascan) in the time domain. This kind of correlation presents a similar sensitivity to that of TDOCT systems (hence 20dB lower than FDOCT). As compared to FDOCT the Ascan is directly registered (and hence displayed) in the time domain whereas in FDOCT the acquisition is realized in the spectral domain. Several systems are possible using different possible configurations and interesting properties have been demonstrated for spectroscopic purpose and for dispersion compensation [36-40]. The system used in this work is described in Fig2.

The experimental set-up used is composed of two parts.

(i) Firstly the sample information is encoded via an interferometer. The latter is illuminated with a supercontinuum of light issued from a microstructured optical fibre pumped by a Q-switched Nd-YAG laser (by Leukos Innovative Optical Systems). The Supercontinuum has a full spectral range from $350 \mathrm{~nm}$ up to $2300 \mathrm{~nm}$. However in experiments reported here, the effective bandwidth is of $150 \mathrm{~nm}$ (Full Width at Half Maximum) centred around $625 \mathrm{~nm}$. Therefore, in a medium with a 
refractive index of 1.5 , such as cellulose, the axial resolution is about $1 \mu \mathrm{m}$. A part of the light is focused on the sample with a telecentric lens. The size of the beam on the sample is about $15 \mu \mathrm{m}$. The light beams issued from the interferometer are directed toward transmission diffraction grating (528 lines $/ \mathrm{mm}$ ) disposed in the perpendicular direction. Because of the incident angles of the two beams, the transverse direction of the diffraction grating introduces a time-delay varying linearly between the recombined beams. The sample depth is thus encoded across the grating that forms a time correlation axis.

(ii) The second part of the set-up is primarily an imaging system that forms the image of the diffraction grating on a two dimensional CCD image sensor (8 bits, $1256 \times 1024$ pixels, 20 frames per second in binning mode). Then the lines of the CCD camera encode the depth of the sample and an A-scan is obtained without scanning along the image lines.

The total recorded signal is the sum in intensity of individual fringe patterns formed by each wavelength of the incident beams. Two temporal signals $r(t)$ and $s(t)$ with the same polarization state are incident onto the grating $(\mathrm{G})$ with the same angle and opposite sign. $r(t)$ and $s(t)$ have complex spectrum given by $\hat{R}(v)$ and $\hat{S}(v)$ respectively where $\hat{R}(v)$ and $\hat{S}(v)$ are Fourier transforms of $r(t)$ and $s(t)$. They also can be expressed with their complex form as

$\hat{R}(v)=R(v) e^{j \cdot \varphi_{R(v)}}$

$\hat{S}(v)=S(v) e^{j \cdot \varphi_{S(v)}}$

with $v$ the frequency of the signal.

We consider in the following one diffracted beam ( -1 order) the other one being only transmitted by the diffraction grating (zero order). The modulus of the spectrum of the diffracted beam is directly accessible in the spectral plane of the spectrometer which is physically in the back focal plane of the lens L. The other beam that is simply transmitted by the grating is focused at a single point in the same back focal plane. Based on upper considerations a simple analysis leads to the expression of total intensity $C(x)$ in the plane D:

$C(x)=I_{0}+2 \operatorname{Re}\left[\int \hat{R}(v) \hat{S}(v) e^{-j 2 \pi\left(\frac{x}{\gamma c}\left(2 \sin \theta_{i}\right)\right) v} e^{j .2 \pi \frac{x}{\gamma \Lambda}} \cdot d v\right]$

where $\mathrm{z}$ is the horizontal coordinate along the CCD camera lines, $I_{0}$ the background intensity, $R e$ denotes the real part. $\Lambda$ is the grating period, $\gamma$ the magnification of the 
imaging system, $\theta_{i}$ the incident angle of the beam and $c$ the speed of light in vacuum. The reference beam $\hat{R}(v)$ is assumed to be real while $\hat{S}(v)$ can be either real or complex depending on the optical sample properties.

The reader may refer to reference [36-40] for a complete overview of such systems. The image acquisition is done with NI Labview Software.

\subsection{Focused Ion Beam}

During the last decade, the use of dual beams, i.e. Focused Ion Beam (FIB) and Scanning Electron Microscope (SEM), has become widespread in the fields of materials and micro-nanotechnology because it offers on the same platform the capability to image and mill locally a wide range of materials and objects [44] with a very high resolution. Two equipments were used to conduct the experiments as we started to work on a SEM LEO 440 upgraded with a FIB column Orsay Physics Canion 31 (L440) which was then replaced by a FEI Helios Nanolab 600i (FEI600i). As the fibres are composed of organic material, a thin layer of chromium $(100 \mathrm{~nm})$ has been deposited at the surface of the fibres by sputtering. This layer has two main goals: to allow a good electrical conductivity to avoid charge effects, and to protect the fibre during the milling process.

The first experiments conducted on the L440 are divided into two main steps: at first, the fibre is cut with a high probe current $(600 \mathrm{pA})$ and then the part of the fibre to be observed is polished with a lower probe current $(60 \mathrm{pA})$ in order to get the minimum surface roughness. The shown images have been performed with the FIB probe instead of the SEM probe because it allows an enhanced contrast (Fig. 3a).

Most of the FIB experiments shown in this paper have been made thanks to the FEI600i (see sketch on figure). The position used to cut the fibre is the position 2 . The first step consists in the deposition in situ of a $1 \mu \mathrm{m}$ thick layer of Pt to protect the area of interest and to avoid the curtain effect. Then, the fibre is cut with the FIB at a high voltage of $30 \mathrm{kV}$ with a high probe current (63 nA) and finally polished at lower probe currents $(9 \mathrm{nA}$ and then $230 \mathrm{pA})$. The SEM allows to see the result after milling, without changing the position of the fibre (for Figs. 3c, $4 \& 5 a$ ). The last experiment (Fig. 5b) is performed on the position 1. The fibre is milled with a $80 \mathrm{pA}$ probe current and snapshots are made every 20 s thanks to the secondary electrons emitted during FIB irradiation. 


\section{Results and discussion}

\section{Internal structure revealed by FIB cutting}

The isolated single fibres were firstly examined using polarised light microscopy to determine their outer diameters, detect the presence of twists or defects in fibre and to check that only a single fibre was present on each paper frame. Indeed, the most common method used in the "plant fibre community" to check the single character of the fibre is to observe it from different angles using polarized light microscopy.

Among these fibres, several fibres with the same order of magnitude in diameter (approximately $30 \mu \mathrm{m}$ ) were selected and prepared for FIB cutting. Some examples of the transverse sections of isolated fibres revealed by the FIB cutting are proposed in Fig. 3b. and 3c. The chromium layer covering the fibres is easily visualized on this picture. Contrary to all expectations, these observations also revealed two types of internal structure:

(i) single fibre with a very thick wall and a small lumen,

(ii) bundle of very small fibres in diameter (from 3 to $11 \mu \mathrm{m}$ ).

The morphology of the fibres has to be described with great care since the experimental conditions used to mill the cell wall (vacuum, sputtering...) may induce many changes in morphology and can in particular induce the collapse of the fibre. The extent of the collapse may be such that the two sides of the inner wall touch completely closing the lumen. This is particularly evident on several fibres in the bundle on Fig. 3c. Some damages are also obvious. The small "tunnels" in the radial direction (Fig. 3b) corresponding to the ion direction can be explained by a difference of irradiation sensitivity of the wall material in the cross-section of the fibre. These tunnels were also observed by Domenges and Charlet [19].

Anyway, the internal organisation is significantly different between the two fibres. In all likelihood and according to the work of Blake et al. [8], it seems that the first type corresponds to a single primary bast fibre and the second one to a bundle of secondary bast fibres. According to literature [5-9], the diameter of the hemp fibres lies between 2$3 \mu \mathrm{m}$ for the smaller secondary fibres to $40-50 \mu \mathrm{m}$ for the bigger primary fibres. This order of magnitude is consistent with the dimensions of the observed fibres (Fig. $3 \mathrm{~b}$ and $3 \mathrm{c})$. The separation process used for these fibres seems insufficient to reach a complete 
degradation of interfaces between secondary fibres. The chemical composition and/or the ease of access for the degradation agents in middle lamellae are certainly different for primary and secondary fibres.

This result is of great importance for the mechanical characterization of the bast hemp fibres. Indeed, this means that during the test campaigns, the batch of isolated fibres prepared on the mounting tabs, is undoubtedly composed of both single primary fibres and bundles of secondary fibres. This could participate in the high scattering in tensile behaviour and properties observed on hemp fibres. To the best of the authors' knowledge, the potential difference in mechanical behaviour and properties between the two types of fibre has not been properly investigated or characterized in literature. In a bundle of fibres, the interfaces between the ultimate fibres (middle lamellae) and the discontinuities of the fibres along the bundle length could presumably induce some differences in the mechanical behaviour, properties and damage kinetics in comparison to an isolated single fibre. In addition to this structural consideration, it is also possible that single primary and single secondary fibres have basic differences in mechanical behaviour. Pickering et al. [10] shown that the average tensile strength of hemp bast fibres changed during the flowering stage of the plant. They attributed this variation to the change in proportion in primary and secondary fibres and considering that the primary fibres have a higher strength. Taking into account these results and the differences in the fibre morphologies and biochemical composition [5], it seems realistic to believe that primary and secondary fibres expose different mechanical behaviour and properties.

To investigate and quantify properly the potential differences in mechanical behaviour between single primary fibres and bundles of secondary fibres, experimenters need to identify each type of fibres among jumble fibres, as delivered by producers. FIB cutting is not a rational option for this type of tests, since tensile campaigns are generally performed on several hundreds of fibres. FIB cutting is highly time consuming and really expensive. In a next part of this work we propose an optical alternative.

After FIB cutting, we can also observe on the transverse section the different layers constituting the fibre wall (Fig. 4). In addition to the middle lamellae, it clearly appears that the cell wall is made of two main layers. From the external part of the fibre to the lumen, the first one has a thickness of several microns and the second one a few hundred nanometres in thickness. These layers, or more exactly the interface between 
these layers, are emphasised when the fibre is irradiated with $\mathrm{Ga}^{+}$ion beam current during a prolonged time. Fig. 5 shows several images recorded on a same fibre at different time of exposure. Some parts of the cell wall are less sensitive to the $\mathrm{Ga}^{+}$ions irradiation. The identification of these highly resistant constitutive polymers requires some additional experiments, relying on biochemical screening, or prior biochemical knowledge.

370 FIB cuttings were also performed every $50 \mathrm{~nm}$ in the fibre length on a fibre of approximately 30 microns in external diameter. The first cutting revealed that the fibre was a bundle of twelve elementary fibres (Fig. 6a). After each cutting, a secondary electrons image was recorded. The movie made of these pictures is proposed in the animation (Online Resource 1, "FIB.mpg"). Fig. 6a shows recorded images corresponding to three positions in the fibre length (initial point, $10 \mu \mathrm{m}$ and $18 \mu \mathrm{m}$ away from the initial point). The movie and selected images show that both the inner shape and outer shape of the elementary fibres significantly change as the function of the length, even when considering a small fibre length. In fig. 6a, the ultimate fibre numbered 1 is emphasized by highlighting the middle lamella by a thick white line. The evolution of the contour line as a function of the fibre length is obvious. These pictures also show some damages in the cells wall, such as cracks (Fig. 6a. $z_{0}+10 \mu \mathrm{m}$ ). They can originate from experimental conditions or from drying. They can also have been induced during the plant growth or during fibre processing. The main lateral dimensions (along $\mathrm{x}$ and $\mathrm{y}$ directions) of the ultimate fibre $\mathrm{n}^{\circ} 1$ are also plotted as a function of the fibre length (see Fig. 6b). The fibre width increases from 9.1 to $11 \mu \mathrm{m}$ along the y axis and decreases from 4.4 to 3.4 along the $\mathrm{x}$ axis. In this latter direction, a significant increase in width is observed (up to $5.6 \mu \mathrm{m}$ ) in the portion of the fibre where the main damages where observed (near $\mathrm{z}_{0}+10 \mu \mathrm{m}$ ).

As for X-ray, the FIB images recorded after FIB cutting can be used to produce 3D digital representations of elementary fibres by mathematically reconstructing projection data.

\section{Investigation of the internal structure using OCT}

Approximately 10 fibres were also examined using OCT. Intensity profiles were plotted at different positions in the length of the fibre. The normalized intensity in the plane was 
plotted as a function of the fibre thickness. The theoretical intensity vs. thickness plot is represented for a perfectly cylindrical and homogeneous material in Fig. 7. This technique is able to reveal the interface between two media with different optical properties (such as air and fibre in this example). The amplitude of the peak gives the local reflectivity hence it indicates the difference level in refractive indexes between different layers. The width of the peak is normally connected to the spectral bandwidth of the light source. Theoretically, in the case of a Gaussian light source this width is given by $\delta z=\frac{2 \cdot \ln 2 \cdot \lambda^{2}}{\pi \cdot \Delta \lambda}$ if the dispersion of the medium can be neglected where $\lambda$ depicts the central wavelength of the light source, $\Delta \lambda$ its optical bandwidth. In realistic cases $\delta \mathrm{z}$ is broadened as the refractive index of a medium is a function of the wavelength, this effect is called the medium dispersion. This broadening could be used in some cases to access to the physical composition of the medium itself [42]. The distance between the peaks $(\mathrm{x})$ is proportional to the fibre diameter (d) and to the refractive index of the material $\left(\mathrm{n}_{f}\right)$, as written in the following equation:

$x=2 . n_{f} \cdot d$

For plant fibres, the relationship between the thickness and the optical properties is more difficultly established. If considering the fibre cylindrical and the layers concentric, we obtain:

$x=2 \times \sum_{i=1}^{n} 2 . n_{i} \cdot e_{i}+2 . n_{\text {lumen }} \cdot d_{\text {lumen }}$

with $e_{i}$, the thickness of the $i^{\text {th }}$ layer, $n_{i}$ : the refractive index of this layer.

The previous results obtained with FIB cutting have shown that the hemp bast fibres are not perfectly cylindrical and that the layers are not concentric. The layers are also different in composition [2]. The S2 layer is mainly composed of cellulose and hemicelluloses. The middle lamella is also made up of lignin and pectins. The cellulose is well known to birefringent. The cellulose microfibrils angle (MFA) also varies from one layer to another. So considering these differences both in composition and microstructure, it is plausible that the optical properties significantly vary according to the layers. For this type of configuration and geometry, the validity of Eq. 5 can be questioned, and a quantitative approach requires theoretical developments. So, for this reason and for the following results, a qualitative approach is proposed, the thickness axis is dimensionless. 
OCT intensity profiles of two of the tested fibres are plotted in Fig.8. Even if the external aspect observed with Polarised Light Microscopy (Fig. 8, on left) and the external diameters are quite similar for these two fibres, the OCT profiles are really different (Fig. 8, middle). The first profile has two major peaks $\left(\mathrm{M}_{1}\right.$ and $\left.\mathrm{M}_{2}\right)$ and two minor peaks $\left(\mathrm{m}_{1}\right.$ and $\left.\mathrm{m}_{2}\right)$. The second profile is more complex and made up of three series of peaks with varying intensities. This result indicates that further interfaces between different materials are present in the second fibre. After OCT examination, the fibres were transversely cutting at the corresponding specific position in length in order to associate the OCT intensity profile to the internal structure of the fibre at this position. Fig. 8 (right) shows the secondary electron images of the transverse sections of both fibres after FIB cutting. These images clearly show that the first fibre is an elementary fibre (probably a primary bast fibre) and that the second one is a bundle of 8 elementary fibres of a few microns in diameter or width (probably secondary bast fibres). It is also obvious that the OCT technique is able to relate these differences in the internal structure of the fibres. For the first fibre, the most intensive peaks $\left(M_{1}\right.$ and $\left.M_{2}\right)$ are attributed to the interface between the environmental air and the external surface of the fibre. The two other peaks $\left(\mathrm{m}_{1}\right.$ and $\left.\mathrm{m}_{2}\right)$, with a normalized amplitude of approximately 0.2 and 0.3 are attributed to the interfaces between the internal surface of the fibre and the air or medium in the lumen. Effectively, it is well known that the lumen may contain nitrogenous residue of protoplasm or organic/inorganic salts [43]. For the second fibre, the intensity plot is more complex than for a single fibre. The numerous peaks are related to the high number of interfaces between the cell wall and the lumen of the constitutive elementary fibres. The glance of the OCT intensity profiles can easily be used to discriminate the type of fibres.

\section{Conclusion}

This paper has investigated the internal structure and morphology of hemp bast fibres using destructive and non-destructive techniques, i.e. FIB microscopy and OCT.

Results shown for a same range of external diameters (of about 20-30 micons), two types of internal structures: (i) elementary fibres with a thick wall and a small lumen and (ii) bundle of small fibres with an external diameter of a few microns. According to data of literature, these two structures were identified as being respectively primary fibre and bundle of secondary fibres. This result is of great importance for the 
mechanical characterization of the bast hemp fibres. Indeed, this means that during the test campaigns, the batch of isolated fibres is undoubtedly composed of both single primary fibres and bundle of secondary fibres. It certainly participates to the high scattering in results.

We also demonstrate in this paper that OCT, a non-destructive and non-invasive technique, is able to quickly and easily discriminate these types of internal structures.

OCT is also a promising technique to distinguish the sub-layers of the cell wall. Using scans according to different material directions and the development of mathematical algorithm, OCT is also a promising technique able to deliver the 3D morphology of the fibre.

\section{Acknowledgements}

This work was partly supported by the French RENATECH network and its FEMTOST technological facility 


\section{References:}

1. Faruk O, Bledzki A, Fink HP, Sain M (2012) Biocomposites reinforced with natural fibers: 2000-2010. Prog Polym Sci 37:1552-1596

2. Placet V, Trivaudey F, Cisse O, Guicheret-Retel V, Boubakar L (2012) Diameter dependence of the apparent tensile modulus of hemp fibres: a morphological, structural or ultrastructural effect? Compos Part A 43(2):275-287

3. Summerscales J, Dissanayake NPJ, Virk AS, Hall W (2010) A review of bast fibres and their composites. Part 1-Fibres as reinforcements. Compos Part A 41:1329-1335

4. Summerscales J, Dissanayake NPJ, Virk AS, Hall W (2010) A review of bast fibres and their composites. Part 2-Composites. Compos Part A 41:1336-1344

5. Crônier D, Monties B, Chabbert B (2005) Structure and chemical composition of bast fibers isolated from developing hemp stem. J Agric Food Chem 53:8279-8289

6. Hernandez A, Westerhuis W, van Dam JEG (2007) Microscopic study on hemp bast fibre formation. Journal of Natural Fibers 3(4):1-12

7. Mediavilla V, Leupin M, Keller A (2001) Influence of the growth stage of industrial hemp on the field formation in relation to certain fibre quality traits. Ind Crop Prod 13:49-56

8. Blake AW, Marcus SE, Copeland JE, Blackburn RS, Knox JP (2008) In situ analysis of cell wall polymers associated with phloem fibre cells in stems of hemp, Cannabis sativa L. Planta 228:113

9. Sankari HS (2000) Comparison of bast fibre yield and mechanical fibre properties of hemp (Cannabis sativa L.) cultivars. Ind Crop Prod 11:73-84

10. Pickering KL, Beckermann GW, Alam SN, Foreman NJ (2007) Optimising industrial hemp fibre for composites. Compos Part A 38:461-468

11. Aslan M, Chinga-Carrasco G, Sorensen BF, Madsen B (2011) Strength variability of single flax fibres. J Mater Sci 46:6344-6354

12. Virk AS, Hall W, Summerscales J (2010) Failure strain as the key design criterion for fracture of natural fibre composites. Comp Sci Tech 70:995-999

13. Burgert I, Gierlinger N, Zimmermann T (2005) Properties of chemically and mechanically isolated fibres of spruce (Picea abies [L.] Karst.). Part 1: Structural and chemical characterization. Holzforschung 59:240-246

14. Burgert I, Frühmann K, Keckes J, Fratzl P, StanzlTschegg S (2005) Properties of chemically and mechanically isolated fibres of spruce (Picea abies [L.] Karst.). Part 2: Twisting phenomena. Holzforschung 59:247-251

15. Burgert I, Eder M, Frühmann K, Keckes J, Fratzl P, StanzlTschegg S (2005) Properties of chemically and mechanically isolated fibres of spruce (Picea abies [L.] Karst.). Part 3: Mechanical characterisation. Holzforschung 59:354-357

16. Bourmaud A, Morvan C, Baley C (2010) Importance of fiber preparation to optimize the surface and mechanical properties of unitary flax fiber. Ind Crop Prod 32:662-667

17. Placet V, Cisse O, Boubakar L (2012) Influence of environmental relative humidity on the tensile and rotational behavior of hemp fibres. Journal of Materials Science 47(7):3435:3446

18. Abbey B, Eve S, Thuault A, Charlet K, Korunsky A (2010) Synchrotron X-ray Tomographic Investigation of Internel Structure of Individual Flax Fibres. IFMBE Proceedings 31:1151-1154

19. Domenges B, Charlet K (2010) Direct insights on flax fiber structure by focused ion beam microscopy. Microsc Microanal 16(2):175-182

20. Charlet K, Jernot JP, Eve S, Gomina M, Bréard J (2010) Multi-scale morphological characterisation of flax: From the stem to the fibrils. Carbohyd Polym 82:54-61

21. Clair B, Gril J, Baba K, Thibaut B, Sugiyama J (2005) Precautions for the structural analysis of the gelatinous layer in tension wood. IAWA Journal 26(2):189-195 
22. Koivu V, Turpeinen T, Myllys M, Timonen J, Kataja M (2009) Three dimensional single fibre imaging in micro- and nano-scales. Proceedings of the Workshop on Single Fiber Testing and Modeling. The Paper Mechanics Cluster and COST Action FF0802

23. Malek M, Khelfa H, Poilane C, Mounier D, Picart P, Investigation of dynamic properties of linen fiber with digital holographic tomography, Forum on Volume Reconstruction Techniques for 3D Fluids \& Solid Mechanics, Poitiers, France, November 29-December 01, 2011

24. Fujimoto JG, Pitris C, Boppart SA, Brezinski ME (2000) Optical Coherence Tomography: An Emerging Technology for Biomedical Imaging and Optical Biopsy. Neoplasia 2(1-2):9-25

25. Morgner U, Drexler W, Fujimoto JG (1999) In vivo ultrahigh-resolution optical coherence tomography. Opt. Lett. 24:1221-1223

26. Huang D, Swanson EA; Lin CP, Schuman JS, Stinson WG, Chang W, Hee MR, Flotte T, Gregory K, Puliafito CA, Fujimoto JG (1991) Optical Coherence Tomography. Science 254:1178-1181

27. Hettinger JW, de la Pena Mattozzi M, Myers WR, Williams ME, Reeves A, Parsons RL, Haskell D, Petersen C, Wang R, Medford JI (2000) Optical coherence microscopy. A technology for rapid, in vivo, non-destructive visualization of plants and plant cells. Plant physiol 123(1):3-15

28. Reeves A, Parsons RL, Hettinger JW, Medford JI (2002) In vivo Three-dimensional imaging of plants with optical coherence microscopy. J microsc 208(3):177-189

29. Fercher AF, Hitzenberger CK, Kamp G, Elzaiat SY (1995) Measurement of intraocular distances by backscattering spectral interferometry Opt. Commn. 117:43-48

30. Leitgeb R, Hitzenberger CK,. Fercher AF (2003) Performance of fourier domain vs. time domain optical coherence tomography. Opt. Express 11:889-894

31. Fercher AF, Drexler W, Hitzenberger CK, Lasser T (2003) Optical coherence tomography principles and applications Rep. Prog. Phys. 66:239-303

32. Drexler W, Fujimoto JG (2008) Optical coherence tomography: technology and applications Springer Verlag.

33. Zeylikovich I, AlfanoRR (1996) Ultrafast dark-field interferometric microscopic reflectometry Opt. Lett.21:1682-1684

34. Verrier I, Brun G, Goure JP (1997) SISAM interferometer for distance measurements Appl. Opt. 36:6225-6230

35. Connes P (1957) Un nouveau type de spectromètre : l'interferomètre réseaux J. Mod. Opt. 4:136-144

36. Froehly L, Ouadour M, Furfaro L, Sandoz P, Gharbi T, Leproux P, Huss G, Couderc V (2008) Spectroscopic OCT by grating-based temporal correlation coupled to optical spectral analysis Int. J. Biomed. Imaging 2008752340

37. Froehly L, Furfaro L, Sandoz P, Jeanningros P (2009) Dispersion compensation properties of grating-based temporal-correlation optical coherence tomography systems. Opt. Commun. 282:1488-1495

38. Froehly L, Iyer S, Vanholsbeeck F (2011) Dual-fibre stretcher and coma as tools for independent 2nd and 3rd order tunable dispersion compensation in a fibre-based scan-free' time domain optical coherence tomography system. Optics Communications 284(16-17):4099-4106

39. Froehly L, Meteau J (2012) Supercontinuum sources in Optical coherence tomography: a state of the art and the application to scan-free time domain correlation techniques and depth dependant dispersion compensation. Opt. Fiber Tech. 18(5):411-419

40. Froehly L, Leitgeb R (2010) Scan-free optical correlation techniques : history and applications to optical coherence tomography J. Opt. 12(8) 084001

41. Volkert CA, Minor AM (2007) Focused ion beam microscopy and micromachining. MRS Bulletin 32(5):389-399

42. Grajciar B., Lehareinger Y., Fercher A., and Leitgeb R. (2010) High sensitivity phase mapping with parallel Fourier domain optical coherence tomography at $512000 \mathrm{~A}$-scan/s. Opt. Express $18,21841-21850$

43. Lewin M., Pearce E.M.(1998) Handbook of fiber Chemistry, Second Edition, Revised and Expanded. CRC Press, International Fiber Science and Technology series/15, New York. 


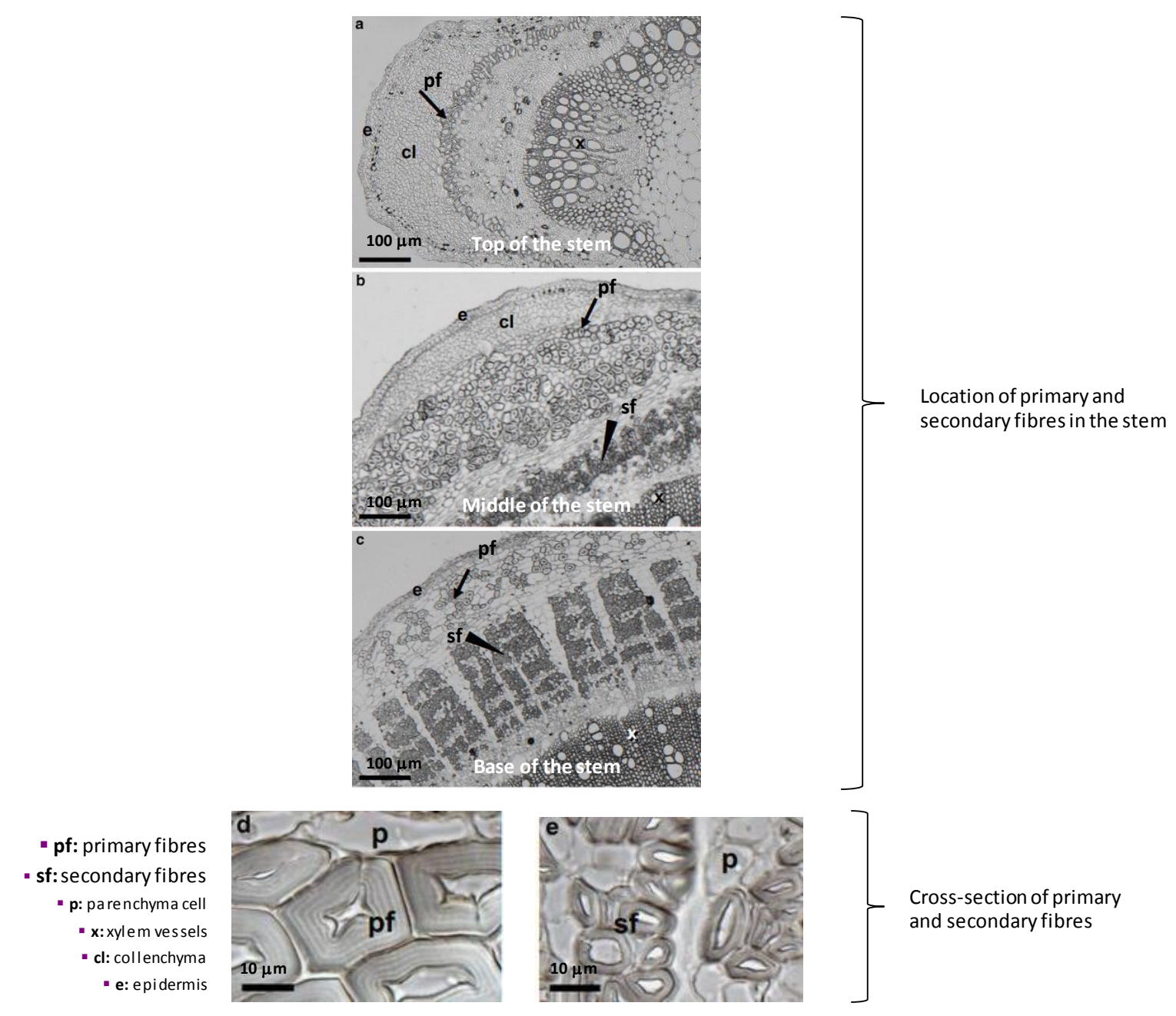

Figure 1: Stem location, morphology and dimensions of primary and secondary bast fibres of hemp.

a-e: Micrographs of transverse sections of hemp stem and hemp bast fibres. From Blake et al. [8] with the kind permission of Paul Knox for use here. 


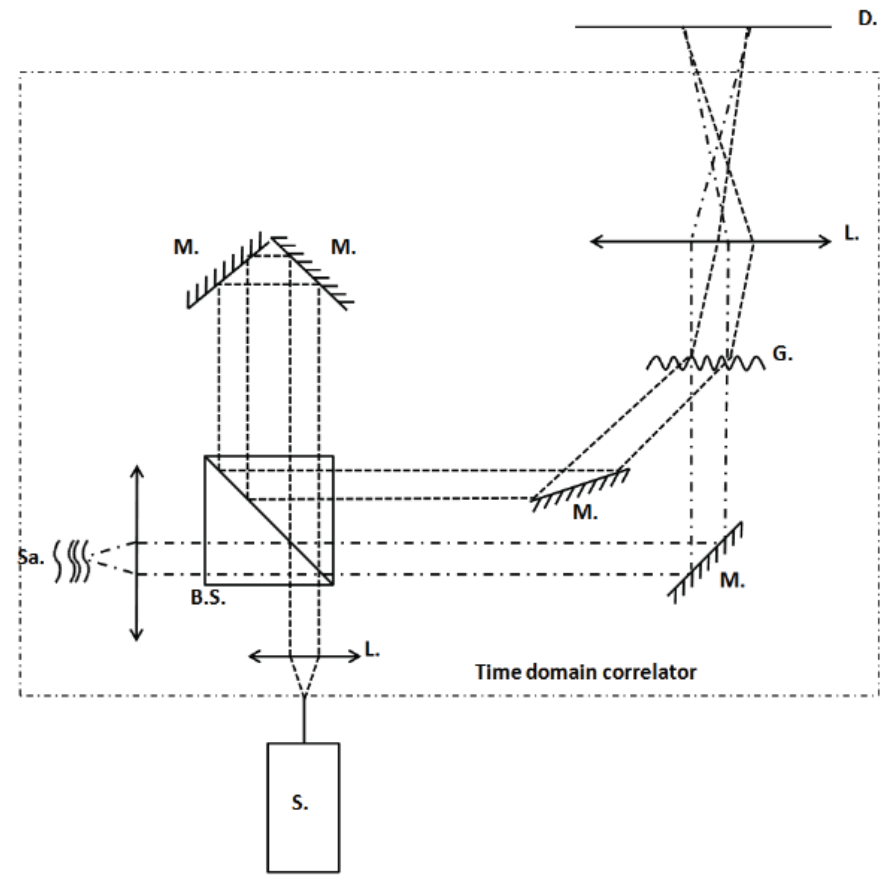

Scan free Time domain correlator : S, supercontinuum laser source ; L, lens ; BS, beam splitter ; Sa, sample ; M, mirror ; G, diffraction grating ; D, CCD detector

Figure 2: Optical Coherence Tomography - Experimental configuration. 


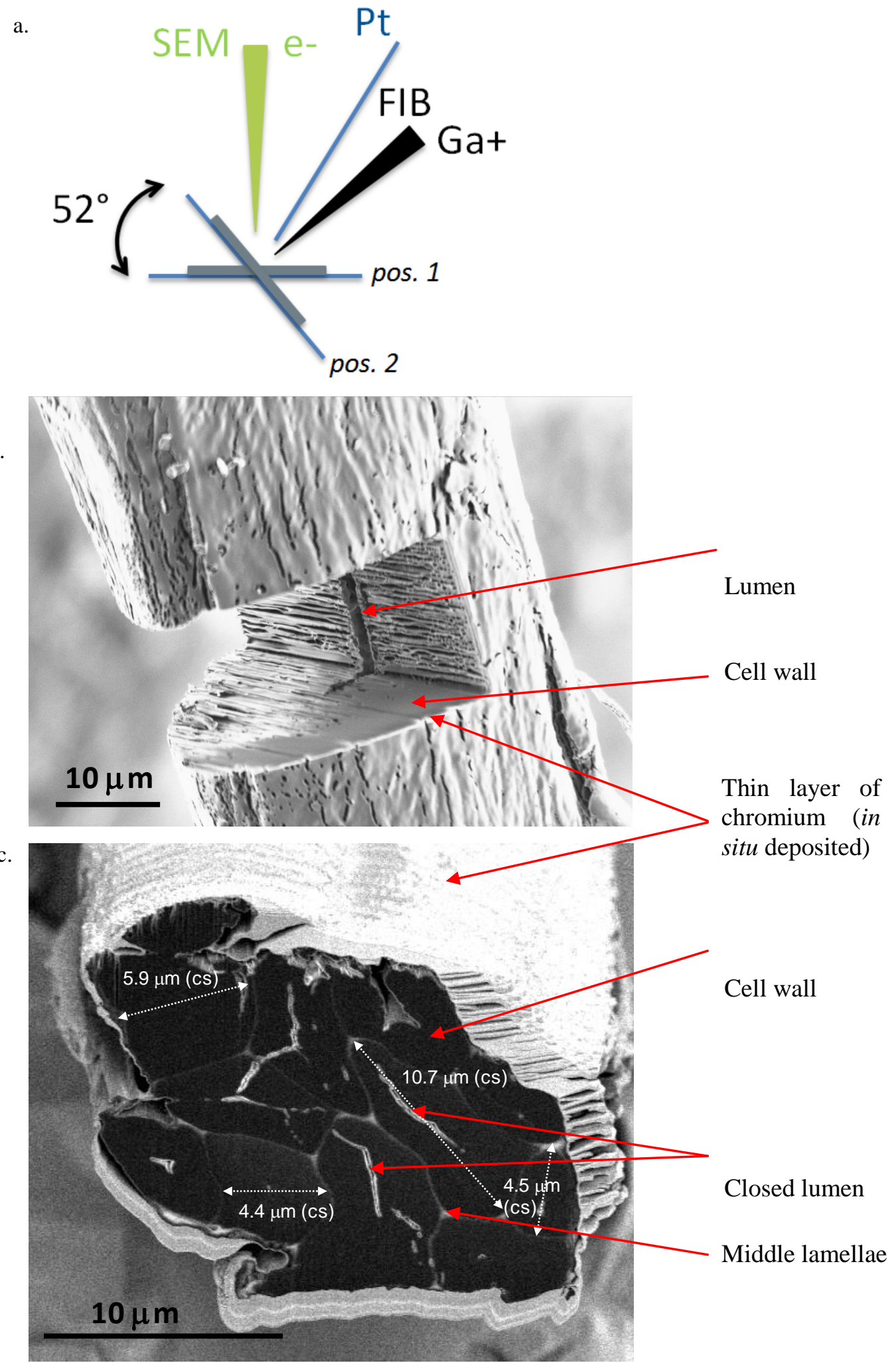

Figure 3: Transverse sections of primary and secondary hemp fibres

a: FIB configuration - dual beam FEI600i.

b: FIB secondary electrons (L440) image $\left(\right.$ tilt $=30^{\circ}$ ) of a isolated and elementary primary fibre with a partial ablation of the cell wall.

c: SEM (FEI600i) image (tilt $=52^{\circ}$ ) of a isolated bundle of secondary fibres after transverse cutting. cs: annotation indicating that measurements include tilt correction 


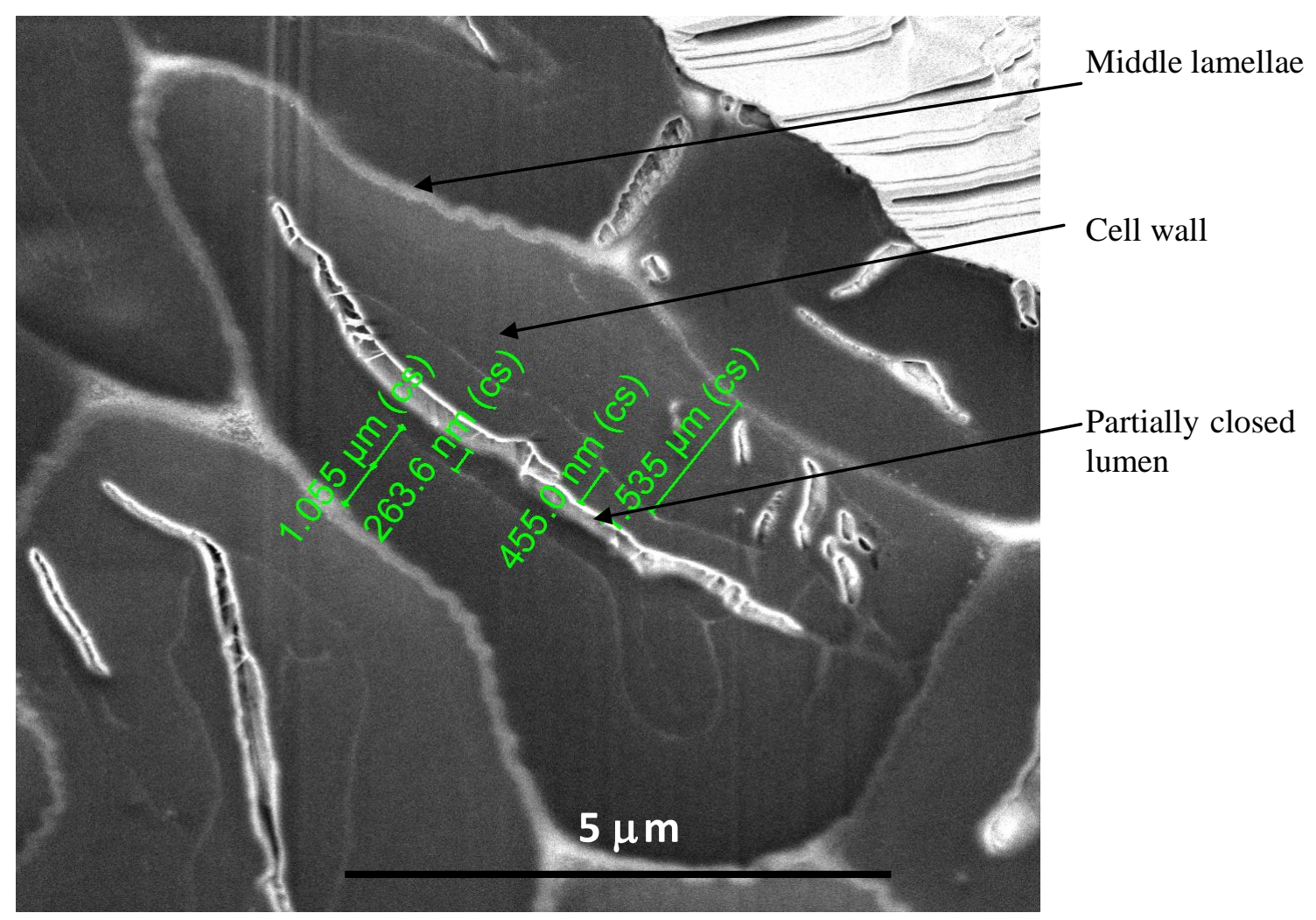

Figure 4 : SEM picture of a hemp fibre $\left(\right.$ tilt $=52^{\circ}$ ).

cs: annotation indicating that measurements include tilt correction 
a.
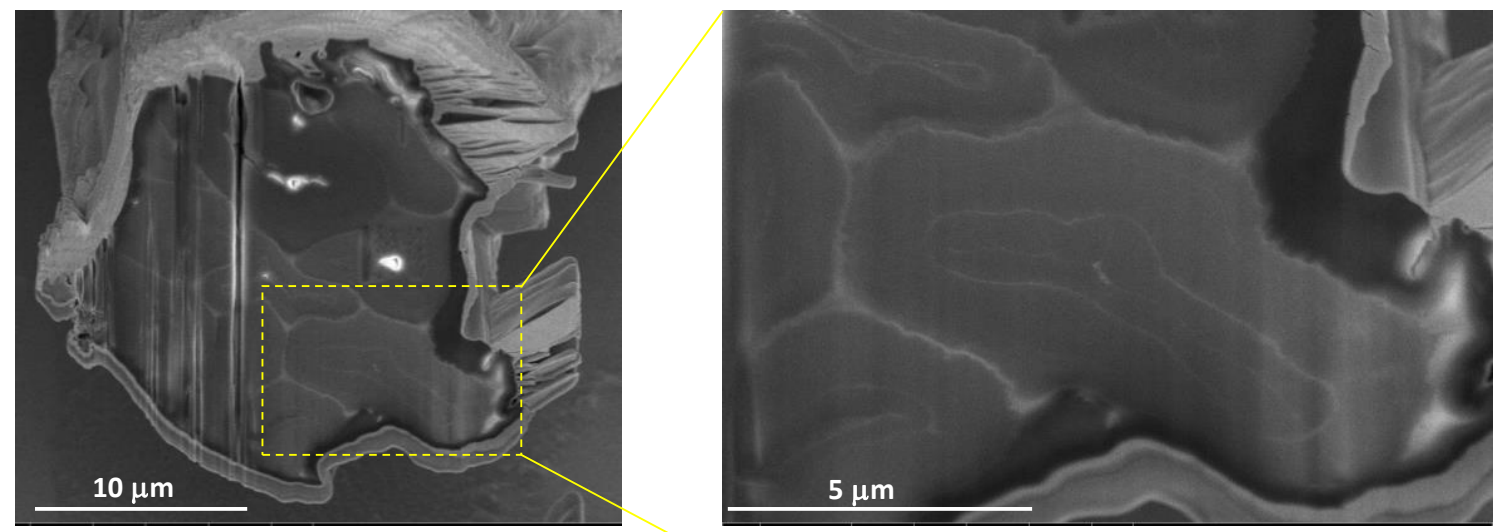

b.
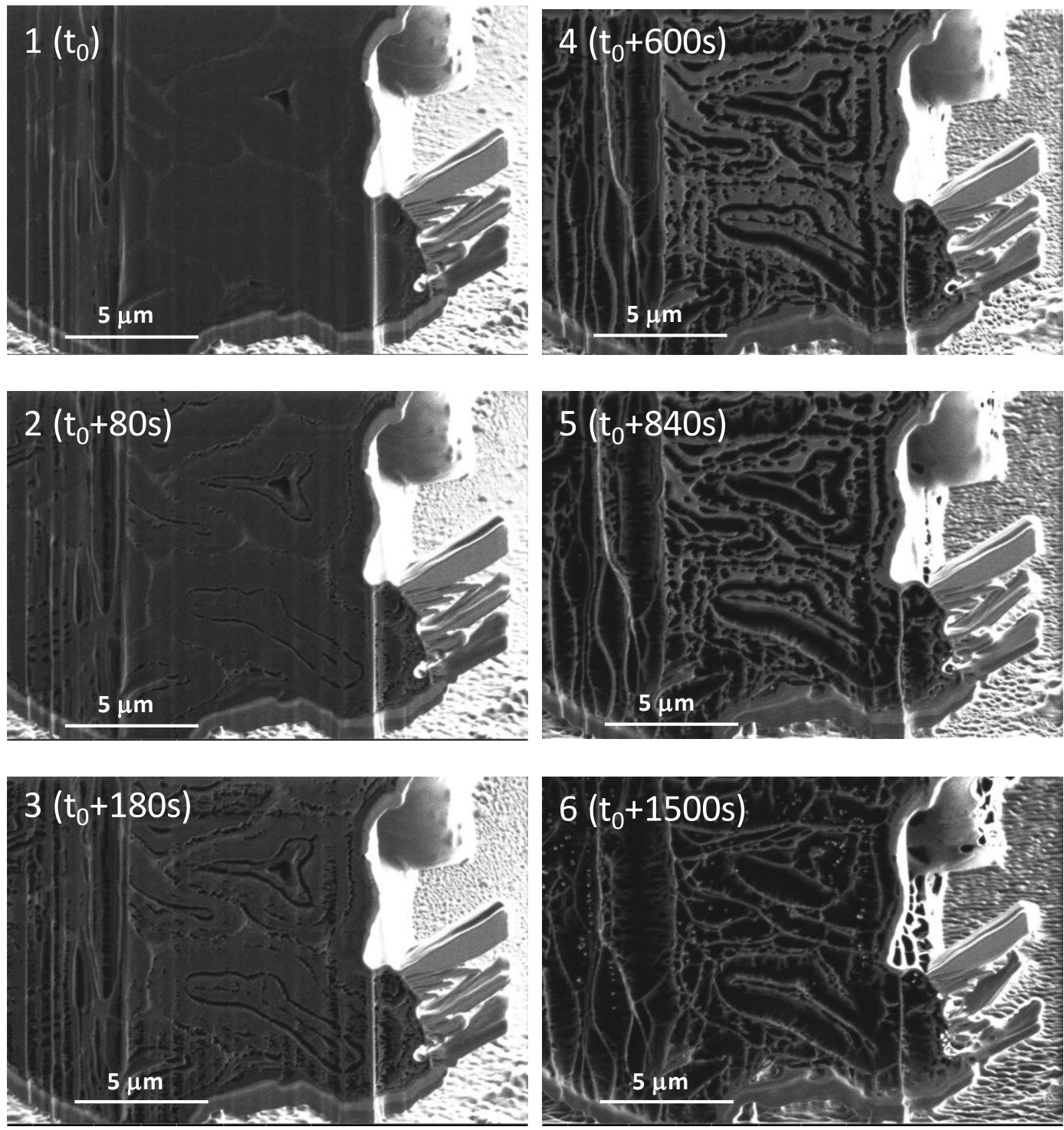

Figure 5 : a. SEM images of a bundle of fibres after FIB cutting. b. FIB secondary electrons images recorded as a function of time exposure to ions after cutting. 
a.
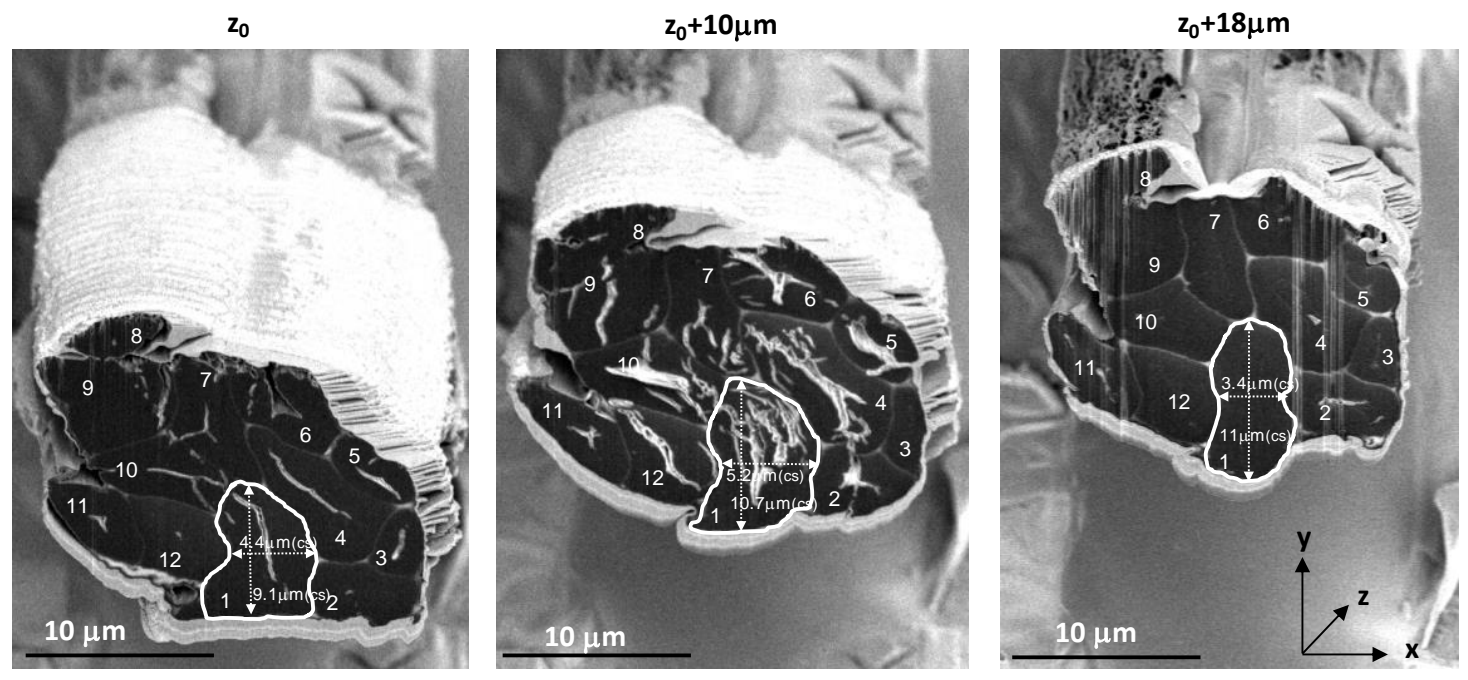

b.

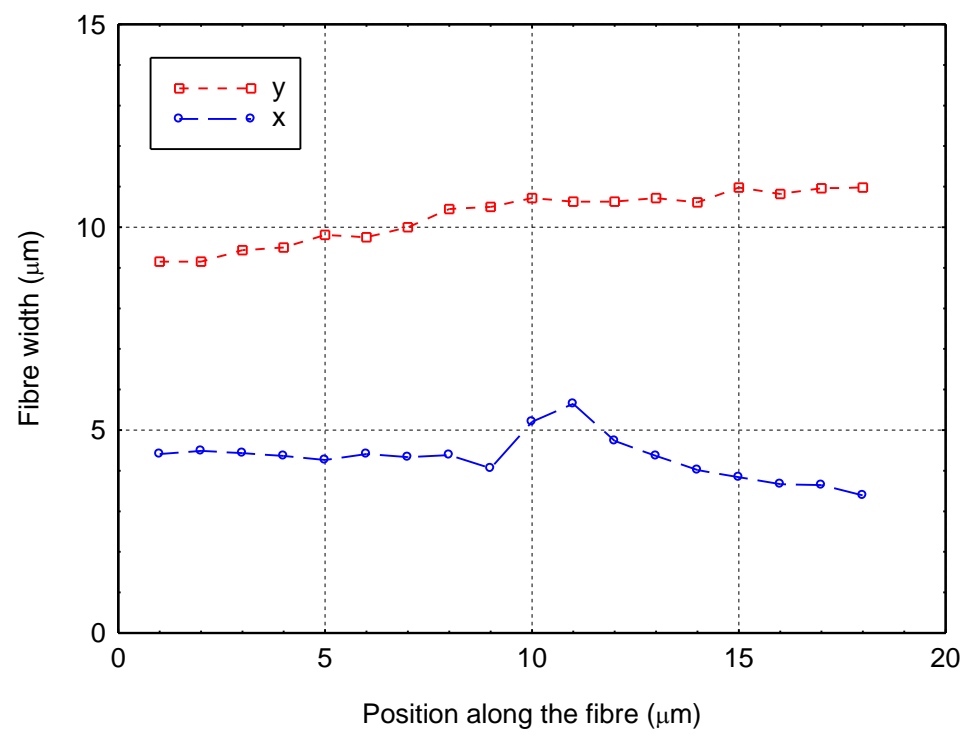

Figure 6: a. SEM (FEI600i) image $\left(\right.$ tilt $=52^{\circ}$ ) of a isolated bundle of 12 secondary fibres after transverse cutting at different positions in the fibre length $\left(z_{0}, z_{0}+10 \mu \mathrm{m}\right.$ and $\left.z_{0}+18 \mu \mathrm{m}\right)$. b. Evolution of the widths (along $x$ and $y$ direction) of the ultimate fibre $n^{\circ} 1$ as a function of the position along the fibre.

cs: annotation indicating that measurements include tilt correction 

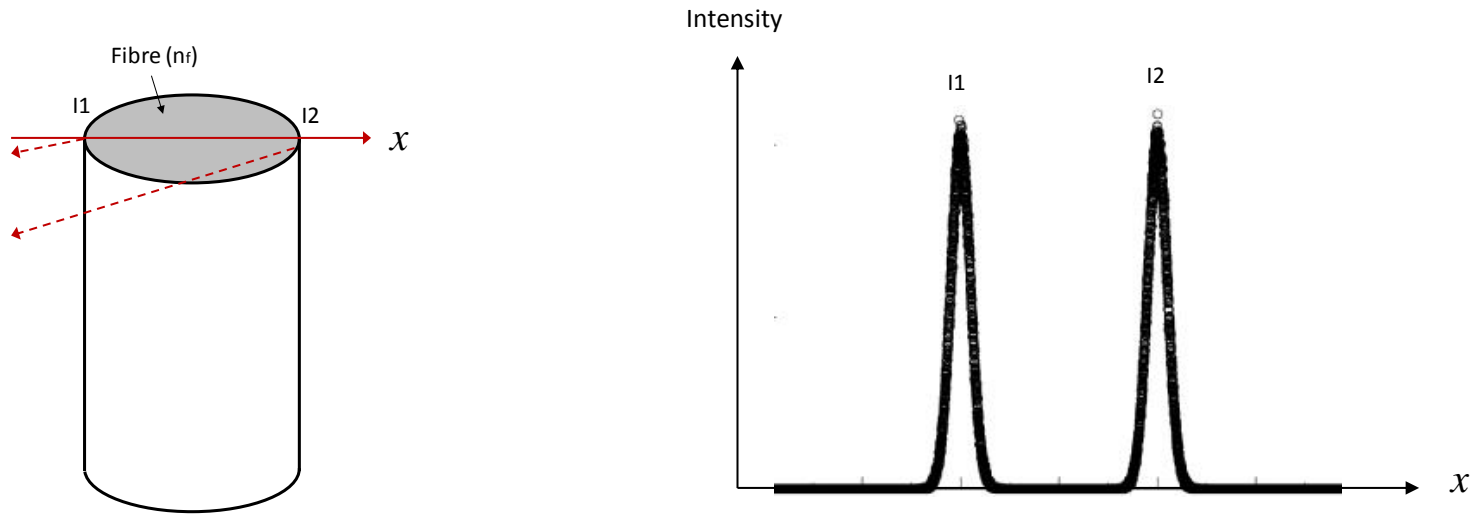

Figure 7: Schematic representation of OCT applied to a perfect cylindrical and homogeneous fibre. 

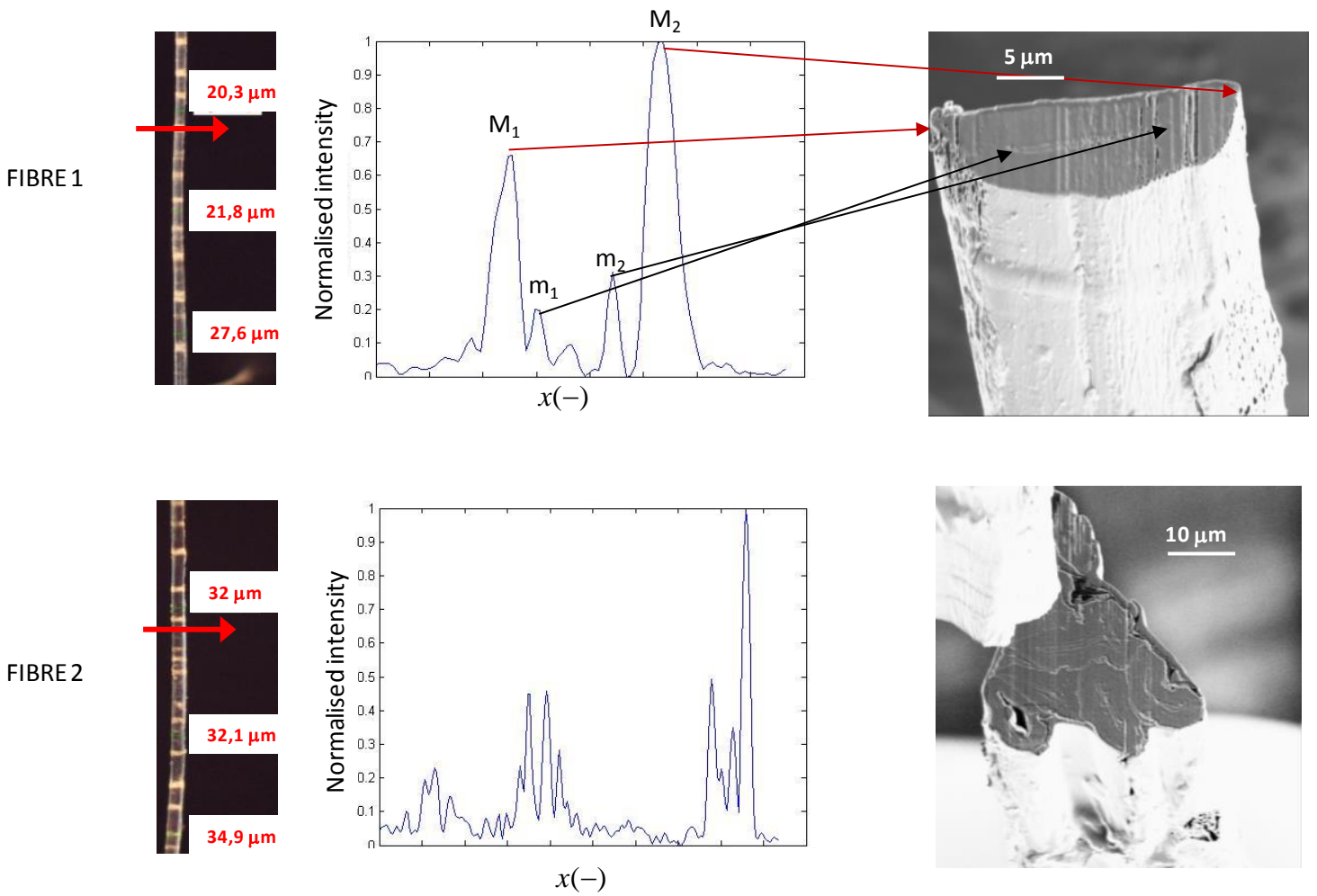

Figure 8: Comparison of OCT intensity profiles of two fibres with different internal structures. Left: Optical observations (Polarised Light Microscopy), Middle: OCT intensity profiles of fibre at the cutting level (before coating and cutting), Right: SEM image of the cross-section of fibres after cutting. Results show that the internal structure of fibres can be characterized using OCT. 